\title{
LECTURE
}

\section{Circuit Theory Based on New Concepts and Its Application to Quantum Theory}

\section{Application of Analog Transmission Circuit Theory to Gauge Theory of Quantum Physics}

\author{
Nobuo Nagai ${ }^{1}$ (Hokkaido University) and Takashi Yahagi ${ }^{2}$ (Signal Processing Technology Laboratory) \\ E-mail:1nagai@es.hokudai.ac.jp, 2yahagi@risp.jp
}

\begin{abstract}
The strong and weak interactions in quantum theory represent the physical phenomena that bind quanta. We attempt to apply impedance matching that represents the connection of circuit elements in analog circuit theory to these interactions. The interactions in quantum theory are related to the phase in gauge theory. The phase can be given to the connected state of circuit elements using cascade matrices, which can determine the phase of circuits. We show that asymmetric LC ladder circuits are suitable for representing the interactions of quanta in terms of the phase as well as the quantized wave equations. We also show that the equivalent circuits for the connection of quarks and gluons can be represented as LC ladder circuits.
\end{abstract}

Keywords: strong interaction, weak interaction, analog circuit theory, impedance matching, gauge theory, phase, cascade matrix, asymmetric LC ladder circuit, quanta of connected quark and gluon, quantized wave equation

\section{Introduction}

In Session 19, we showed that two quanta are bound to form another quantum using the quantized wave equations. Therefore, it is considered that circuit theory can be applied to explain the phenomenon that, for example, neutrons and protons are bound to form atomic nuclei.

It is understood that objects are electromagnetically bound by the attraction of electrically positive and negative charges and that objects are attracted to each other by the attractive force. However, the binding of nucleons in an atomic nucleus is unclear, although it is partly explained by gauge theory and is known to be related to the strong and weak interactions.

In this session, we assume that the formation of quanta such as atomic nuclei is represented by the quantized wave equations. Circuit theory is applied to this assumption, where the phase can be defined. Thus, we attempt to apply circuit theory to the strong and weak interactions.

\section{Strong and Weak Interactions}

To examine the strong and weak interactions in quantum theory using the quantized wave equations, we cite sentences related to "God's Corner-Cutting" and part of the ending of "Charm Quarks Must Exist" in Chapter 18 of "Inclined Framework of Weak Interaction" in Ref. [1]:

'God's Corner-Cutting: The weak interaction is difficult to understand. This is because the binding constant is small and the distance of interaction is short, but these are not the only reasons. Other interactions, such as gravitation, electromagnetism, and the strong interaction, are explained within the framework of gauge theory and follow the law of symmetry, whereas the weak interaction is very irregular and seems to have no complete symmetry. I have increasingly considered that the weak interaction is caused by not only the parity (P) violation, charge-parity (CP) violation, and strangeness violation but also other factors. I will explain what I always feel when considering the problem of the weak interaction.'

When God designed the universe, He made accurate drawings for the gravity, electromagnetic force, and strong force by following some formulae. However, God might have made a mistake in calculating the weak force or reading its scale, resulting in the generation of contradictions in some parts of its drawing. Hence, straight lines do not intersect perpendicularly and quadrilaterals do not form correctly. The framework of the weak force is slightly inclined with respect to those of the other forces, 
but this is not distinctive if seen from far away. Therefore, God used the incorrect framework as it is and constructed the universe.

However, scientists believe that the Almighty God never carried out such an irresponsible deed and attempt to explain any phenomena. They consider that the inclination of the framework is for a certain reason rather than a careless mistake.

Owing to the tremendous effort over about 20 years, our understanding of the weak interaction has greatly advanced. The weak interaction was organized and uniformly described by Weinberg-Salam theory. Ultimately, it has become possible to conclude that the weak interaction follows the principle of gauge fields similarly to the other interactions.'

'Charm Quarks Must Exist: It is an unsolved mystery why the framework of the weak interaction is inclined with respect to that of the other interactions. However, it is also interesting to imagine the world if there was no inclination and the design by God was perfect. All the generations of quarks and leptons would have identical properties excluding mass, and unless they were mixed, each generation would have a conserved quantity. For example, even if c (charm quark) was converted into s (strange quark) in the second generation, s could not be converted into $u$ (up quark) in the first generation. Therefore, the lambda $(\Lambda)$ particles and $\mathrm{K}$ mesons would be stable, and atomic nuclei would consist of protons, neutrons, $\Lambda$ particles, and so forth, resulting in a world quite different from the present world. Living organisms might not have existed. The design of the inclined framework might have been intended by God.'

The above part of Ref. [1] can also be interpreted as follows. The binding of quanta related to quarks excluding leptons is represented by the strong and weak interactions. These interactions are difficult to explain but are uniformly described by gauge theory. As described in Ref. [2], gauge theory was developed from the idea of a mathematician, $\mathrm{H}$. Weyl, and the phase is the most basic physical quantity in the theory. According to Ref. [3], the phase in gauge theory can be extended to superstring theory.

In the next section, we attempt to apply circuit theory to the phase in gauge theory. First, the phase in gauge theory is examined using the quantized wave equations.

\section{Analog Circuit Theory and Digital Circuit}

The main purpose of this session is to apply circuit theory to the phase in gauge theory. Before starting this discussion, we examine circuit theory in more detail.

The strong and weak interactions in quantum theory are the interactions that bind quanta. From the standpoint of applying the Maxwell equations and circuit theory as a field of electric science to quantum theory, the interactions in quantum theory bind quanta, that is, "connect" quanta, and are thereby considered to correspond to impedance matching in circuit theory.
Circuit theory up to and including the 1960s meant analog circuit theory. Currently, advances in artificial intelligence (AI) technologies are leading to the development of a highly information-based society, which was brought about by the rapid progress of digital technologies using computers. Digital circuit technologies have also made rapid progress but do not include the concept of impedance matching. Therefore, impedance matching is a manifestation of the old analog circuit theory.

Here, let us review the history of circuit theory on the basis of Ref. [4]. Circuit theory progressed as a result of innovative inventions and discoveries such as the development of a calculation method for electric circuits developed by Kirchhoff and the introduction of $j \omega$ for alternating current phenomena by Steinmetz. The scientist who perhaps made the greatest contribution to the development of circuit theory is Heaviside. According to Ref. [4], Heaviside boldly developed an operational calculus to convert differential equations to algebra. The operational calculus was combined with complex function theory to develop realization theory based on positive real functions proposed by Brune. A widely used concept in circuit theory at least 50 years ago was Darlington synthesis, summarized in Ref. [5]. Moreover, the operational calculus strongly influenced the development of Euclidean space, Hilbert space, and generalized function theory (distribution or hyperfunction) in mathematics [4].

Despite his contribution to the development of mathematics, Heaviside was ignored by mathematicians [6]. This may be because he believed that physical phenomena that cannot be treated by rigorous mathematics exist in circuit theory; indeed, he once said, "Rigorous mathematics is narrow, physical mathematics bold and broad" [6]. As a representative problem treatable by circuit theory, he explained that a cable using an inductive loading coil is a distortionless transmission line. However, Heaviside wrote that when he first suggested inductive loading, a wellknown physicist, Thomas Blakesley, said "it would be like making humps on a road to increase the speed of vehicles."

As Blakesley considered, reflection occurs when a coil is loaded in a transmission line, resulting in the disruption of transmission. This idea is related to digital circuits, and no reflection is assumed to occur in digital circuits. In contrast, the inductive loading concept proposed by Heaviside is innovative for analog circuits, as described in Sessions 1 and 13. Circuits have transient and steady states, which provide different responses. Digital circuits determine an instantaneous waveform and do not assume reflection. For analog circuits, the determination of a steady-state response is emphasized and reflection can be assumed in a transient state. It is enough that impedance matching takes place in a steady state. Thus, Heaviside was considered to have focused on steady-state responses.

Heaviside reformatted Maxwell's equations with scalars and vector potentials as basic quantities into equations with two complex quantities, that is, complex voltage and 
complex current, as basic quantities. These were derived from the telegraphers' equations. Here, we discuss a few aspects of the telegraphers' equations that play an important role in analog circuits.

The telegraphers' equations are expressed as a onedimensional uniform line. As the solutions of its eigenvalue problem, voltage and current are determined, enabling Maxwell's equations to be handled on the basis of circuit theory. The telegrapher's equations are partial differential equations expressed with time $t$ and position $x$. To express the partial differential equations using the circuit elements L, $\mathrm{C}, \mathrm{R}$, and $\mathrm{G}$, the function part of time $t$ is expressed as $\exp (j \omega t)$ by variable separation or Laplace transform, which is explained by the operational calculus proposed by Heaviside [6]. That is, the equations are easily explained by assuming that the time variable is expressed by $\exp (j \omega t)$ in circuit theory, similarly to $j \omega$ adopted by Steinmetz in alternating current phenomena. Although waves and vibrations with an angular frequency of $\omega$ are expressed by $\sin \omega t$ or $\cos \omega t$ in conventional circuit theory, the use of $j \omega$ by Steinmetz allows waves and vibrations to be expressed as complex vibrations using $\exp (j \omega t)$.

In electromagnetic theory, energy in electromagnetic fields is defined by momentum. In circuit theory, the time integral of complex power, a product of voltage and current, is defined as energy to examine the law of energy conservation using two complex functions, voltage and current. In relation to this definition, many references explained reactive power; for example, the time integral of reactive power as the imaginary part of complex power is 0 and the reactive power is unrelated to energy. However, voltage and current as the complex functions are obtained after a Laplace transform, and the time variable $t$ is not included in these functions. For analog circuits, there are transient and steady states, and power is divided into active and reactive powers in a steady state. It is considered appropriate to define the time integral of active power as energy. Mathematically, Fourier analysis and Hilbert space theory are considered to ignore the part that becomes 0 by time integration. However, some cases such as resonance require the examination of reactive power and an analytic method that does not ignore the reactive power. In this case, cascade matrices are useful in circuit theory.

The telegraphers' equations represent transmission lines, and reflection and transmission take place there. When these phenomena are applied to circuit theory to enable the introduction of the reflection and transmission of lines to circuits with different elements and the use of reflected waves in analog circuits, resonance and impedance matching can be used. Note that resonance cannot be achieved in two- and three-dimensional circuits because distributed in-phase waves need to come together for resonance but the transmitted waves diffuse in two- and three-dimensional spaces and the reflected waves cannot return to their original positions. There are some differences between the reflection and transmission in transmission lines and those in circuit elements. That is, the reflection and transmission in transmission lines are expressed by a function of time $t$, whereas those in circuit elements are expressed in a steady state. The reflection and transmission in quantum mechanics are phenomena in quanta rather than lines and thereby expressed in a steady state similarly to circuit elements.

It is considered that Heaviside had difficulty in explaining steady-state responses because the circuit elements produced by the manufacturing technologies in his day had a large loss compared with those produced by current technologies. In contrast, quantum mechanics is considered to be lossless physics because it assumes movements in a vacuum space without friction.

As above, analog circuits are handled using functions of time $t$ on the basis of the operational calculus with a Laplace transform rather than the equation of motion in Newtonian mechanics. Sometimes analog circuits are mysterious from a viewpoint of physics. Thus, analog circuit theory can be applied to the weak interaction described in Section 2. That is, circuit theory is considered to be applicable to gauge theory.

\section{Transmission Problem of Active Power in Analog Circuit Theory}

Figure 20.1 is a circuit diagram showing how to use a circuit whose main purpose is to transmit energy. The transmission of energy via a transmission circuit requires a power source as an energy source and a load that receives the power, as shown in Fig. 20.1(a).
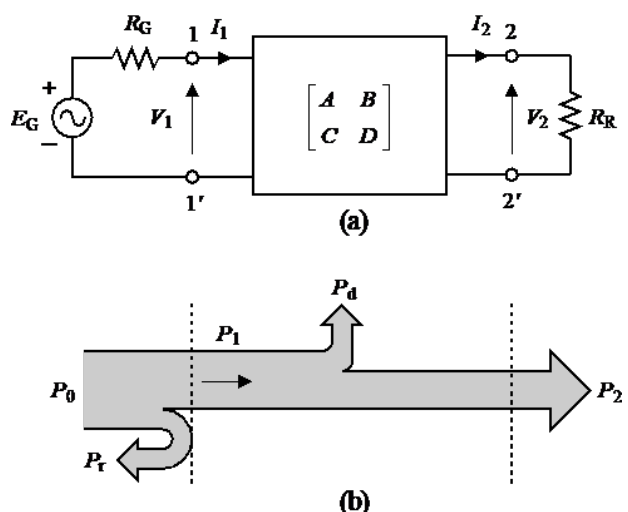

Fig. 20.1 (a) Transmission line to examine law of energy conservation and (b) flow of active power in the circuit

Here, we use a voltage source as an input power source and assume that the voltage with a single angular frequency of $\omega$ is expressed by a complex number $E_{G} \exp (j \omega t)$. That is, only the single angular frequency is input unlike the cases of sinusoidal and cosine functions, and the amplitude of the voltage is given by not an effective value but a complex 
number $E_{G}$ based on the wave height. $E_{G}$ has information on the phase similarly to usual phasers.

The law of energy conservation in circuit theory explains how much and where the energy supplied from a power source goes. In concrete, we define the maximum active power supplied from a power source as the maximum available power $P_{0}[4]$ and examine a circuit with the maximum available power as an input wave. For voltage sources, the maximum available power is given by the voltage and internal resistance $R_{G}$ of the voltage source. For the circuit shown in Fig. 20.1(a), it is defined as

$$
\frac{\left|E_{G} \exp (j \omega t)\right|^{2}}{4 R_{G}}=\frac{\left|E_{G}\right|^{2}}{4 R_{G}}
$$

When the active power supplied to a load is equal to the maximum available power, resonance is achieved and the law of energy conservation holds.

We examine how the law of energy conservation is represented in the transmission circuit shown in Fig. 20.1(a). First, the maximum available power of a power source of a circuit consisting of voltage $E_{G}$ and resistance $R_{G}$ is given by $P_{0}=\operatorname{Re}\left(V_{1} * I_{1}\right)$. We denote the active power reflected at interface 1-1' between the power source and the transmission circuit as $P_{r}$, the active power incident to the right from interface 1-1' as $P_{1}$, the active power consumed as a loss within the transmission circuit as $P_{d}$, and the active power transmitted to the load with a resistance of $R_{R}$ through interface 2-2' and consumed there as $P_{2}$. The conservation of the active power is expressed by

$$
P_{0}=P_{r}+P_{1}=P_{r}+P_{d}+P_{2}
$$

Therefore, the incident power is not equal to the transmitted power unless $P_{d}=0$, and the law of energy conservation does not hold when only the input/output ports are viewed. However, if the transmission circuit is lossless, $P_{d}=0$ and thereby $P_{0}=P_{r}+P_{2}$, the energy is conserved between the input and output ports.

Here, we have considered the relationship among powers focusing on active power only. This is because the reactive power remains and does not transmit energy, and it cannot be related to the energy conservation in the steady state.

As above, a lossless circuit is required for transmission circuits so that the law of energy conservation holds in a circuit with an input port, an output port, and a black box (transmission circuit) between the power and the load. Otherwise, active power is consumed in a transmission circuit, and not all of the maximum available power from the power source can be transmitted to the load.

\section{Application of Impedance Matching in Circuit Theory to Interactions in Quantum Theory}

In circuit theory, circuits are represented by voltage and current. However, transmission problems require the determination of active power rather than voltage and current. That is, it is a problem with impedance, the ratio of voltage to current, as a parameter.
To achieve resonance, lossless circuit elements are required for a black-box transmission circuit in the circuit shown in Fig. 20.1(a). Unit elements whose characteristic impedance is given as a real number are lossless, and an analytic method using unit elements for transmission circuits is described in Session 7. When unit elements are used, the analytic method can determine a transient response using wave digital filters (WDFs) [7] or digital lattice filters (DLFs) [8]. During the transient response, the position and momentum of quanta are not considered to be fixed, and their relevant reflected and transmitted waves cannot be specified. Thus, the uncertainty principle in quantum mechanics is considered to correspond to the transient response.

In circuit theory, the impedance of lossless circuit elements is given by a purely imaginary number. Such circuit elements include coils (with an inductance of $L$ ) and capacitors (with a capacitance of $C$ ). In the following sections, we examine the problem of resonance in the case that a transmission circuit has elements with purely imaginary impedance.

\subsection{Series LC resonant circuit}

The circuit shown in Fig. 20.2 is examined. Assuming that the power source is a voltage source with a single frequency and its voltage is $E_{G}$, the internal impedance $Z_{G}$ is a complex number given by

$$
Z_{G}=R_{G}+j X_{G}
$$

The load impedance $Z_{R}$ is also a complex number given by

$$
Z_{R}=R_{R}+j X_{R}
$$

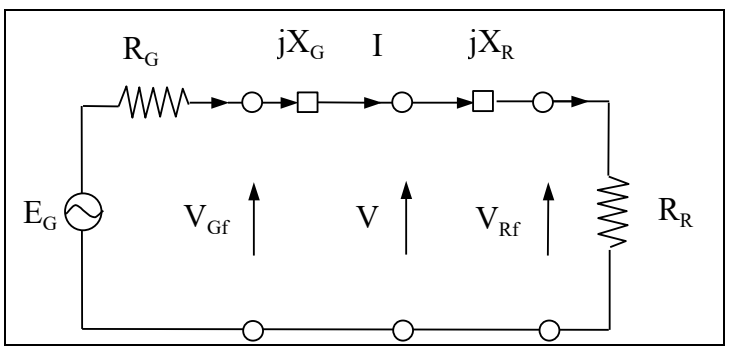

Fig.20.2 Transmission circuit using complex numbers

First, we determine the maximum active power, which is supplied from the power source in the circuit and is the maximum power consumed at a load with resistance $R_{R}$.

The current $I$ flowing through the circuit is given by

$$
I=\frac{E_{G}}{R_{G}+R_{R}+j\left(X_{G}+X_{R}\right)}
$$

Therefore, the complex power $P_{t}$ at a load with resistance $R_{R}$ is given by

$$
P_{t}=\frac{\left|E_{G}\right|^{2} R_{R}}{\left(R_{G}+R_{R}\right)^{2}+\left(X_{G}+X_{R}\right)^{2}}
$$

This is a real number and thereby the active power. 
It is well known that $P_{t}$ takes a maximum when the load impedance is the complex conjugate of the internal impedance of the power source, that is, when the following equations are satisfied:

$$
\begin{aligned}
& X_{R}=-X_{G} \\
& R_{R}=R_{G}
\end{aligned}
$$

At this time, the maximum active power $P_{i}$ is given by

$$
P_{i}=\frac{\left|E_{G}\right|^{2}}{4 R_{G}}
$$

Because this is equal to the maximum available power, resonance is achieved.

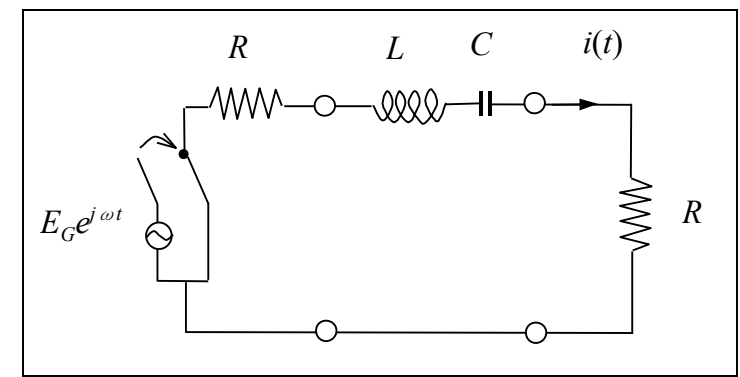

Fig.20.3 Series LC resonant switching circuit

When the purely imaginary part of impedance is positive, the circuit element is assumed to be a coil. When it is negative, the circuit element is assumed to be a capacitor. A circuit that achieves resonance is shown in Fig. 20.3.

The impedances of a coil with inductance $L$ and a capacitor with capacitance $C$ in the circuit shown in Fig. 20.3 are given by

$$
\begin{aligned}
& j X_{G}=j \omega L \\
& j X_{R}=-j \frac{1}{\omega C}
\end{aligned}
$$

The resonance angular frequency $\omega_{0}$ of the circuit is given by

$$
\omega_{0}=\frac{1}{\sqrt{L C}}
$$

In Section 2 of Ref. [9], the transient response of the circuit shown in Fig. 20.3 is determined. In this session, however, the purpose is to clarify the relationship between impedance matching and the phase in gauge theory. We now focus on the phase of the transmission circuit in Fig. 20.3 .

In circuit theory, image and iterative parameters can be obtained from cascade matrices, and the phase is included in the parameters. Here, we attempt to determine the iterative phase. The transmission circuit shown in Fig. 20.3 is a series LC circuit, and its cascade matrix is given by

$$
\left(\begin{array}{ll}
A & B \\
C & D
\end{array}\right)=\left(\begin{array}{cc}
1 & j \omega L-j \frac{1}{\omega C} \\
0 & 1
\end{array}\right)
$$

In Session 6 of this lecture series, we showed that the iterative parameters are derived from the eigenvalue problem of a cascade matrix. The eigenvalue of the cascade matrix given by Eq. (20.9) is obtained from

$$
\left|\begin{array}{cc}
1-\Lambda & j \omega L-j \frac{1}{\omega C} \\
0 & 1-\Lambda
\end{array}\right|=(1-\Lambda)^{2}=0
$$

The eigenvalue obtained from Eq. (20.10) means the iterative phase. However, the eigenvalue is 1 and the iterative phase cannot be determined. This indicates that, in the series LC circuit, resonance can be determined as a transmission problem but the phase cannot be determined. Therefore, the circuit cannot be treated by wave functions. In this session, we call such a circuit a series LC resonant circuit.

\subsection{LC ladder circuit}

Quanta must be bound with each other to form a substance. In this session, we attempt to apply circuit theory to the binding operation and discuss impedance matching.

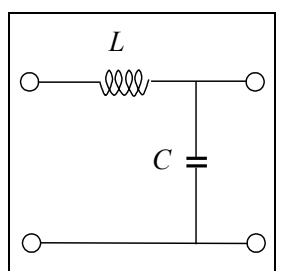

Fig.20.4 Combined basic quantum (Asymmetric LC ladder circuit)

The series LC resonant circuit shown in Fig. 20.3 is suitable for examining impedance matching but cannot be used to determine the iterative phase. Hence, the phase required in gauge theory cannot be introduced. When series $\mathrm{C}$ in the transmission circuit in Fig. 20.3 is assumed to be a separate element, the circuit shown in Fig. 20.4 is obtained. As described in Session 19, this circuit can be obtained from the quantized wave equations. The impedances of the left and right sides are determined from the iterative parameters and are complex conjugates. The circuit satisfies the conditions of impedance matching and the iterative phase is determined.

\section{Future Works}

In the subsequent sessions of this lecture series, we will discuss the application of circuit theory considering the LC ladder circuit shown in Fig. 20.4 as a basic quantum. Gluons bind quarks or are bound with quarks via a strong force. In this session, we assumed that quarks and gluons are paired and equivalently expressed by the LC ladder circuit shown in Fig. 20.4. Neutrons and protons are the quanta compositely bound and their equivalent circuits are considered complicated. However, the equivalent circuits can be approximated by the LC ladder circuit shown in Fig. 20.4. It is assumed that neutrons and protons satisfy the 
quantized wave equations and are bound with other neutrons and protons.

In this lecture series, we attempt to apply circuit theory to quantum mechanics and assume the LC ladder circuit shown in Fig. 20.4 as a basic quantum considering that impedance matching corresponds to a binding operation. In conventional physics, however, physicists observe phenomena as a result of the collision of two particles in an accelerator [1], which sometimes corresponds to a transient response in circuit theory. In physics, the interaction between quanta represented by the Feynman diagram [10] corresponds to a transient phenomenon. Moreover, the magnetic moment of electrons, for which a value with a precision of 10 decimal places or higher is required, is obtained in a steady state. Thus, we feel that steady-state and transient responses are assumed to be identical phenomena in physics. In future work, we examine what results are obtained by discriminating the steady-state and transient responses on the basis of circuit theory.

\section{Acknowledgments}

We are deeply grateful to Professors Kazuhisa Watanabe and Hirofumi Sanada at Hokkaido University of Science for their continued cooperation in our discussion. We are deeply grateful to Professor Hiroshi Tanimoto at Kitami Institute of Technology for his continued cooperation in our discussion via e-mail. We also thank Dr. Jie Ren for her continued support in the development of the figures and calculations.

\section{References}

[1] Y. Nambu: Quark, 2nd edition -How Far Has Elementary Particle Physics Advanced? Kodansha, Ltd., B-1205, 2008. (in Japanese)

[2] A. Tonomura: Watching a Gauge Field, Kodansha Ltd. (Blue Backs, B1162), 1997. (in Japanese)

[3] H. Ooguri: Dr. Ooguri's Introduction to Superstring Theory (Ultimate Theory in Nine-Dimensional World), Bluebacks B1827, Kodansha Ltd., 2013. (in Japanese)

[4] H. Watanabe: Theory and Design of Transmission Networks, Ohmsha, Ltd., 1968. (in Japanese)

[5] Darlington Memorial Issue, IEEE Circuits and Systems, I : Fundamental Theory and Applications, Vol. 46, No. 1, Jan. 1999.

[6] P. J. Nahin: OLIVER HEAVISIDE - The Life, Work, and Times of an Electrical Genius of the Victorian Age - , The Johns Hopkins University Press, 2002.

[7] A. Fettweis : Wave digital filter theory and practice, Proc. IEEE, Vol.74, No.2, pp.270-327, 1986.

[8] A. H. Gray, JR., and J. D. Markel: Digital lattice and ladder filter synthesis, IEEE Trans. Audio Electro-Acoust., Vol. AU21, No. 6, pp.491-500, 1973.

[9] N. Nagai: Circuit Theory Based on New Concept and Its Application to Quantum Mechanics, Seibunsha, 2013. (in Japanese)

[10] R. P. Feynman: QED - The Strange Theory of Light and
Matter-, Princeton University Press, 1985.

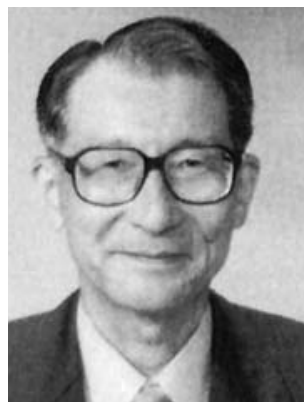

Nobuo Nagai received his B.S. and D.Eng. degrees from Hokkaido University in 1961 and 1971, respectively. In 1961, he joined Hokkaido University as an Assistant and in 1972 he became an Associate Professor, and from 1980 to 1992 he was a Professor in the Research Institute of Applied Electricity. From 1992 to 2001, he was a Professor in the Research Institute for Electronic Science, Hokkaido UniUniversity. In 2001, he retired and became an Emeritus Professor. His research interests are circuit theory and digital signal processing. He is interested in the application of above theory to quantum theory. Dr. Nagai is a Life Fellow of the Institute of Electronics, Information and Communication Engineers, Japan, and a Life Member of IEEE and IEICE, and an Honorary Member of RISP.

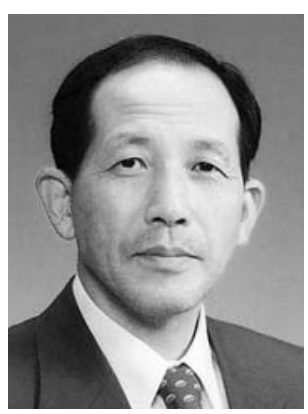

Takashi Yahagi received his B.E., M.S. and Ph.D. degrees all from the Tokyo Institute of Technology in 1966, 1968 and 1971, respectively. In 1971, he joined Chiba University as a Lecturer and in 1974 he became an Associate Professor, and from 1984 to 2008 he was a Professor at the same university. Since 2008 he has been with the Signal Processing Research Laboratory. In 1997, he founded the Research Institute of Signal Processing, Japan (RISP). Since 1997 he has been President of RISP. From 1997 to 2013 he was Editor-in-Chief of the Journal of Signal Processing (JSP). Since 2013 he has been Honorary Editor-in-Chief of JSP. He was the author of "Theory of Digital Signal Processing (Vols. 1-3)", (1985, 1985, 1986), Corona Pub.Co., Ltd. (Tokyo, Japan). He was also the editor and author of "Library of Digital Signal Processing (Vols. 1-10)", (1996, 2001, 1996, 2000, 2005, 2008, 1997, 1999, 1998, 1997), Corona Pub.Co., Ltd. (Tokyo, Japan). He was the editor of "My Research History (Vols. 1 and 2)" (2003, 2003), RISP. The contents of the Library of Digital Signal Processing are as follows: Vol.1: Digital Signal Processing and Basic Theory (1996), Vol.2: Digital Filters and Signal Processing (2001), Vol.3: Digital Signal Processing of Speech and Images (1996), Vol.4: Fast Algorithms and Parallel Signal Processing (2000), Vol.5: Kalman Filter and Adaptive Signal Processing (2005), Vol.6: ARMA Systems and Digital Signal Processing (2008), Vol.7: VLSI and Digital Signal Processing (1997), Vol.8: Communications and Digital Signal Processing (1999), Vol.9: Neural Network and Fuzzy Signal Processing (1998), Vol.10: Multimedia and Digital Signal Processing (1997). Dr. Yahagi is a Life Fellow of the Institute of Electronics, Information and Communication Engineers, Japan. 